\title{
Cutting NiTi with Femtosecond Laser
}

\author{
L. Quintino, ${ }^{1}$ L. Liu, ${ }^{2}$ R. M. Miranda, ${ }^{3}$ R. J. C. Silva, ${ }^{4}$ A. Hu, ${ }^{5}$ and Y. Zhou ${ }^{5}$ \\ ${ }^{1}$ IST-UTL Instituto Superior Técnico, Universidade Técnica de Lisboa, Avenida Rovisco Pais, 1049-001 Lisboa, Portugal \\ ${ }^{2}$ Department of Mechanical Engineering, Tsinghua University, Beijing 100084, China \\ ${ }^{3}$ UNIDEMI, Departamento de Engenharia Mecânica e Industrial, Faculdade de Ciências e Tecnologia, Universidade Nova de Lisboa, \\ 2829-516 Caparica, Portugal \\ ${ }^{4}$ CENIMAT/I3N, Faculdade de Ciências e Tecnologia, Universidade Nova de Lisboa, 2829-516 Caparica, Portugal \\ ${ }^{5}$ Department of Mechanical Engineering, University of Waterloo, 200 University Avenue West, Waterloo, ON, Canada N2L-3G1
}

Correspondence should be addressed to R. M. Miranda; rmiranda@fct.unl.pt

Received 4 May 2013; Accepted 30 July 2013

Academic Editor: Yuanhua Lin

Copyright (C) 2013 L. Quintino et al. This is an open access article distributed under the Creative Commons Attribution License, which permits unrestricted use, distribution, and reproduction in any medium, provided the original work is properly cited.

\begin{abstract}
Superelastic shape memory alloys are difficult to machine by thermal processes due to the facility for Ti oxidation and by mechanical processes due to their superelastic behavior. In this study, femtosecond lasers were tested to analyze the potential for machining NiTi since femtosecond lasers allow nonthermal processing of materials by ablation. The effect of processing parameters on machining depth was studied, and material removal rates were computed. Surfaces produced were analyzed under SEM which shows a resolidified thin layer with minimal heat affected zones. However, for high cutting speeds, that is, for short interaction times, this layer was not observed. A depletion of Ni was seen which may be beneficial in biomedical applications since Ni is known to produce human tissue reactions in biophysical environments.
\end{abstract}

\section{Introduction}

NiTi has been finding increasing applications in industry due to their superior mechanical properties and functional behavior including biocompatibility. As a consequence, $\mathrm{NiTi}$ is being applied in parts and microparts as in stents, sensors, and actuators for biomedics and transport industries.

Microfabrication of these alloys includes material removal, as cutting and drilling, where mechanical processes are difficult to use due to strain hardening experienced by NiTi. High pressure abrasive water jet has also been used for rough cutting operations but is not applicable in high precision cutting since the jet does not guarantee precision and tolerances compatible with those required in microparts. Electric discharge machining (EDM) is feasible but with limited flexibility [1-3].

Lasers are much more adequate, and Nd/YAG lasers have been successfully used. However, they induce a heat affected zone where oxidation of $\mathrm{Ti}$ occurs with depletion of this element and enrichment in $\mathrm{Ni}[4]$ which is detrimental for biomedics. Femtosecond lasers have been exploited due to their accuracy and precision, but mostly because they can remove material by an ablative process in a vapor state with minimal damage of a heat affected zone.

Basically, material decomposition and removal are a consequence of an energy introduced that is above the solid bonding energy. When a very short laser pulse targets a surface, a solid ablation can occur in two stages: absorption by coupling via multiphoton excitation of electrons in the conduction band and energy distribution into the bulk material. Since this diffusion is minimal, a limited amount of liquid phase is formed, and thus material removal occurs mainly by evaporation [5-7]. The liquid film on the irradiated surface resolidifies and can be observed on the cut kerf. For pulse durations below picosecond, absorption is more dominant than the thermal diffusivity, and the heat conduction can be negligible during an ablation process, since evaporation is dominant if the absorbed energy is above the threshold energy for material evaporation $\left(I_{\mathrm{th}}\right)$. The last one is controlled by the specific heat for evaporation $(Q)$, the 


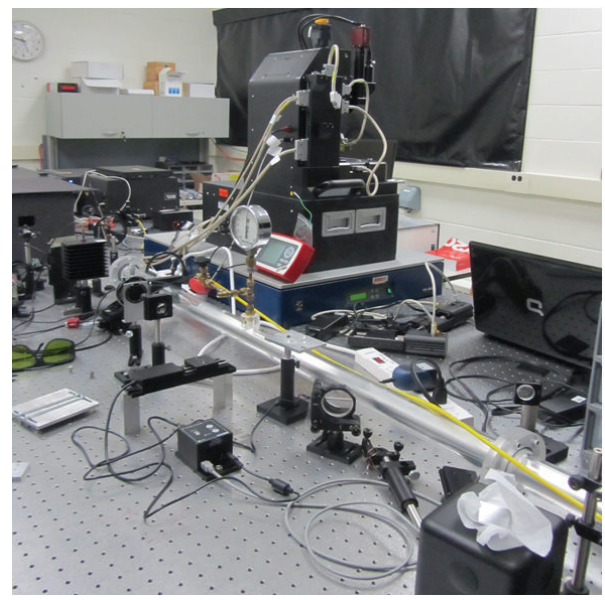

FIGURE 1: Experimental set up: laser head and fixing system.

material density $(\rho)$, the thermal diffusivity $(\alpha)$, and the pulse duration or interaction time $(t)$ as expressed

$$
I_{\text {th }}=\frac{\rho Q}{\alpha t} .
$$

The ablation depth per pulse $(L)$ is, thus, given by (2), and it increases with the laser intensity for a certain material:

$$
L=\frac{I}{\alpha} \ln \left(\frac{I}{I_{\mathrm{th}}}\right) .
$$

Increasing the number of incident pulses coupling is facilitated, and the accumulation of electron excitation with the formation of defects facilitates particle desegregation and material removal.

This study aimed at assessing the effect of processing parameters on the machining depth by computing the material removal rate. The produced surfaces were observed under SEM, and a resolidified thin layer was observed with solidification cracking across the surfaces.

\section{Experimental Approach}

A Ti-sapphire laser system from Coherent Inc. was operated to generate polarized laser pulses with $35 \mathrm{fs}$ duration at a repetition rate of $1 \mathrm{kHz}$ and a peak power of $2.5 \mathrm{~W}$. The laser was emitted at a wavelength of $800 \mathrm{~nm}$ and had a Gaussian beam distribution, and the travel speed was set at $0.01,0.1$ and $1 \mathrm{~mm} / \mathrm{s}$. Laser pulses were focused by a lens with $13 \mathrm{~cm}$ focal length, and a mechanical shutter was used to select the number of pulses. Figure 1 shows the experimental set up adopted. No gas flow was used to assist the cuts.

Machined samples were observed using electron scanning microscopy in backscattered and secondary emitted radiation. Compositional changes resulting from processing were determined by EDS in a Jeol scanning electron microscope (SEM) with an energy dispersive spectrometer (EDS) from Oxford Instruments model INCAx-sight. Semiquantification microanalysis was made using ZAF correction procedure.

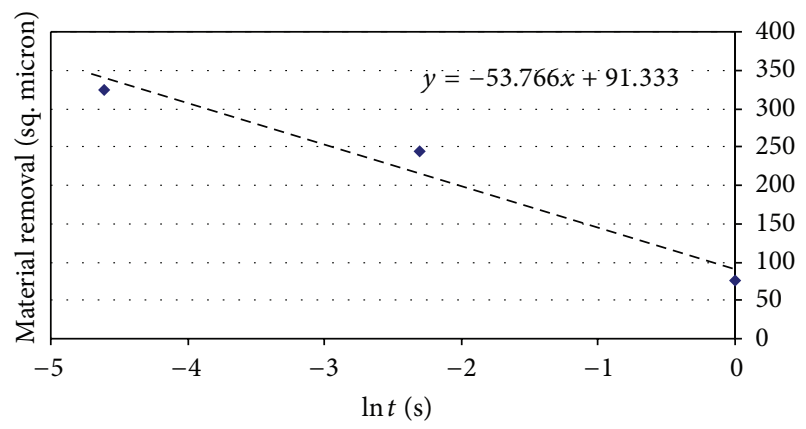

FIGURE 2: Variation of material removal with the logarithm of the interaction time.

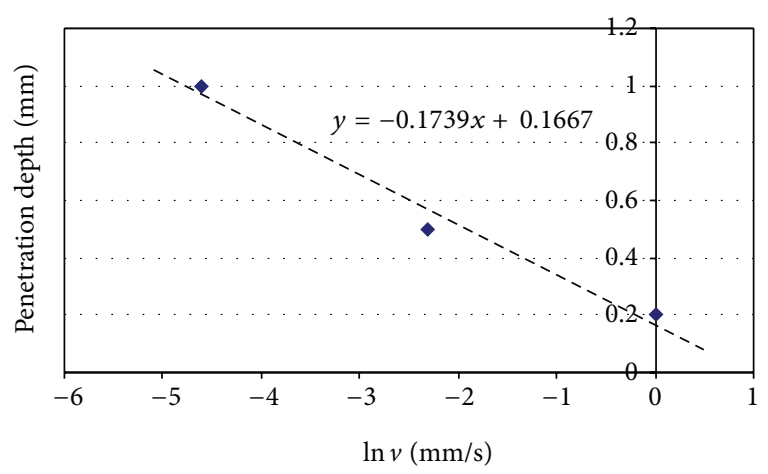

FIgure 3: Penetration depth as a function of cutting speed.

\section{Discussion of Results}

The cross-section of the cuts obtained was analyzed under SEM with EDS and shows that the lower speed led to an ablation A of the total thickness of the sample $(1 \mathrm{~mm})$ while irradiations at lower speeds left lower depth marks. Analyzing the geometry of the surface and with a free image analysis software, the volume of material removed was calculated and plotted as a function of the interaction time as shown in Figure 2 . The interaction time is inversely proportional to the speed, since all other parameters (beam diameter, pulse duration) were kept constant.

This figure shows a linear relationship between the material removal (MR) and the logarithm of the interaction time $(\ln t)$ given by

$$
\mathrm{MR}=91.3-53.8 \ln t
$$

Thus, for NiTi, an empirical equation to calculate the material removal rate can be given by

$$
\mathrm{MR}=A+B \ln t .
$$

This is likely to be applicable for ablation of NiTi with femtosecond lasers. Constants $A$ and $B$ depend on the laser characteristics and processing parameters as laser power output.

The penetration depth has a logarithmic decay with the travel speed as depicted in Figure 3. An empirical equation to 


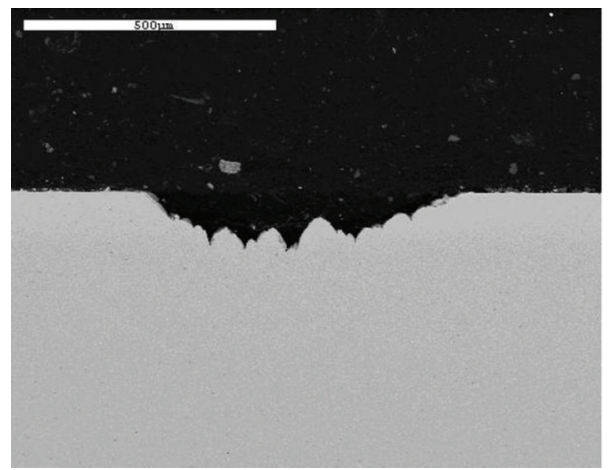

FIGURE 4: Macrograph of sample machined by femtosecond laser $(v=1 \mathrm{~mm} / \mathrm{s})$.

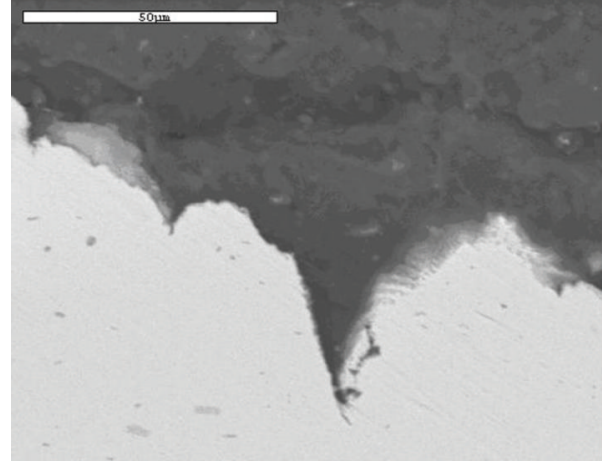

(a)

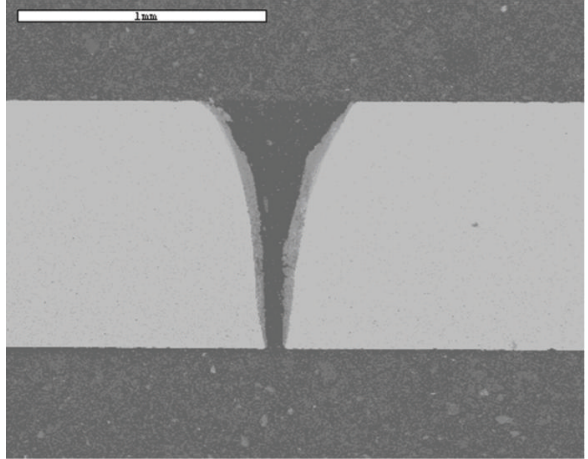

(b)

FIGURE 5: SEM macrographs of cuts with different travel speeds. (a) $v=1 \mathrm{~mm} / \mathrm{s}$; (b) $v=0.01 \mathrm{~mm} / \mathrm{s}$.

determine the penetration depth $(p)$ with the cutting speed (v) can be expressed by

$$
p=0.17-0.17 \ln v .
$$

For $\mathrm{NiTi}$, this proposed equation allows a rough estimation of the penetration achieved with a given cutting speed keeping other processing parameters constant, namely, focus diameter and laser power.

Observing the cut surfaces first under optical microscopy and later under SEM, sharp cuts were seen as shown in Figure 4.

Etching the samples cross-sections, it was not possible to identify any grain boundary structure since this is difficult to reveal in hot worked NiTi; however, there was no noticeable difference near the cut surface, suggesting that the very short interaction time prevented diffusion to occur into the bulk material with grain growth or phase transformations, and thus heat affected zone is inexistent.

Figure 5 shows SEM micrographs of machined surfaces. Complete cut trough was seen when decreasing the travel speed, that is, increasing the number of pulses per interaction area per unit of time.

Observing these samples, a few interesting features can be identified. On the surface of the noncomplete cut, cracked material is seen suggesting a material removal mechanism as described before; that is, the successive laser irradiations coupled with the material induce the formation of cumulative defects that break the material into very small fragments leading to disaggregation. A rippled structure is seen free of redeposits or solidified layers as in the sample cut at lower travel speed. In this case, the energy introduced was well above the threshold for evaporation, and the exceeding heat dissipated into the bulk material, melting a thin layer of the surface. This layer resolidified, and this process is followed by cracking.

Elemental semiquantitative analysis in the base material and in the resolidified zone was performed with EDS. The Xray spectrums are shown in Figure 6.

The solidified layer is oxidized, and both $\mathrm{Ti}$ and $\mathrm{Ni}$ are lost in this oxidation process, with a reduction in $\mathrm{Ni}$.

\section{Conclusions}

From this study, it was seen that

(i) femtosecond lasers can cut NiTi with a high precision and a minimal heat affected zone,

(ii) empirical equations are proposed to calculate the material removal and penetration in ablation of $\mathrm{NiTi}$ with femtosecond lasers within the experimental range used, 


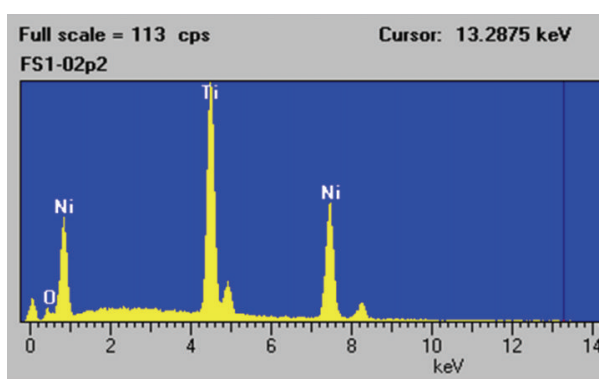

(a)

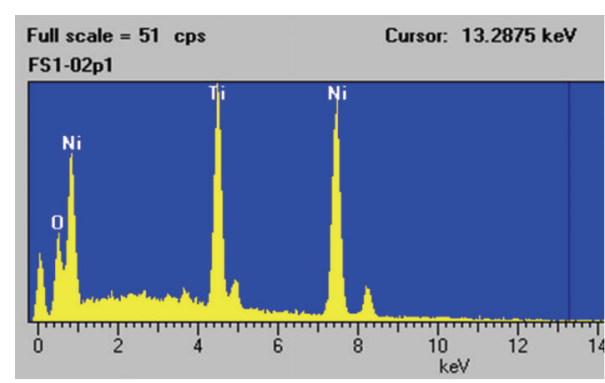

(b)

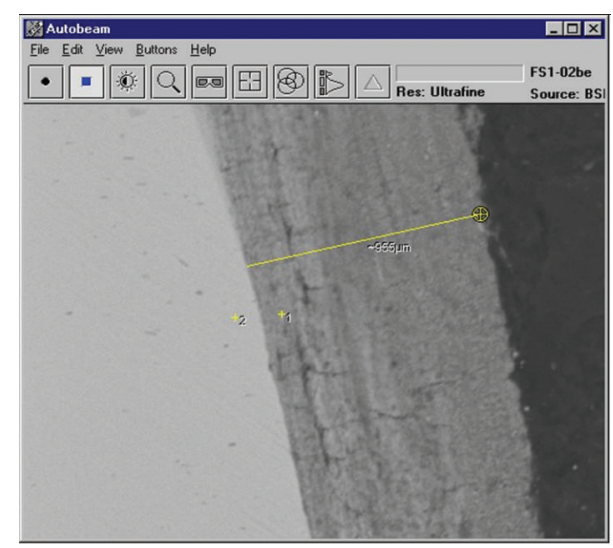

FIGURE 6: SEM view of a cut surface in NiTi with microanalysis. (a) EDS spectrum of bulk material; (b) EDS spectrum in the resolidified layer.

(iii) for low cutting speeds, the surfaces show a resolidified thin layer with solidification cracks and oxidation. This was not observed for high cutting speeds when the interaction time decreased,

(iv) A depletion of $\mathrm{Ni}$ was seen which is beneficial specially in biomedical applications; though biocompatibility tests were required to confirm this statement.

\section{References}

[1] R. M. Miranda, F. M. B. Fernandes, C. M. Craciunescu, L. Quintino, and L. A. Vieira, "Shape memory alloys: existing and emerging applications," in Advances in Materials Science Research, M. C. Wythers, Ed., vol. 6, chapter 7, Nova Science, New York, NY, USA, 2011.

[2] H. C. Lin, K. M. Lin, and Y. C. Chen, "A study on the machining characteristics of TiNi shape memory alloys," Journal of Materials Processing Technology, vol. 105, no. 3, pp. 327-332, 2000.

[3] H. C. Lin, K. M. Lin, and I. S. Cheng, "The electro-discharge machining characteristics of TiNi shape memory alloys," Journal of Materials Science, vol. 36, no. 2, pp. 399-404, 2001.

[4] J. P. Oliveira, F. M. B. Fernandes, and R. M. Miranda, Laser Welded NiTi, LAP, Saarbrücken, Germany, 2013.

[5] H. Hu, X. Wang, and H. Zhai, "High-fluence femtosecond laser ablation of silica glass: effects of laser-induced pressure," Journal of Physics D, vol. 44, no. 13, pp. 135-202, 2011.

[6] H. Huang, H. Y. Zheng, and G. C. Lim, "Femtosecond laser machining characteristics of Nitinol," Applied Surface Science, vol. 228, pp. 201-206, 2004.
[7] J. Reif, "Basic physics of femtosecond laser ablation," in LaserSurface Interactions for New Material Production, A. Miotello and P. Ossi, Eds., Tailoring Structures and Properties, 2010. 

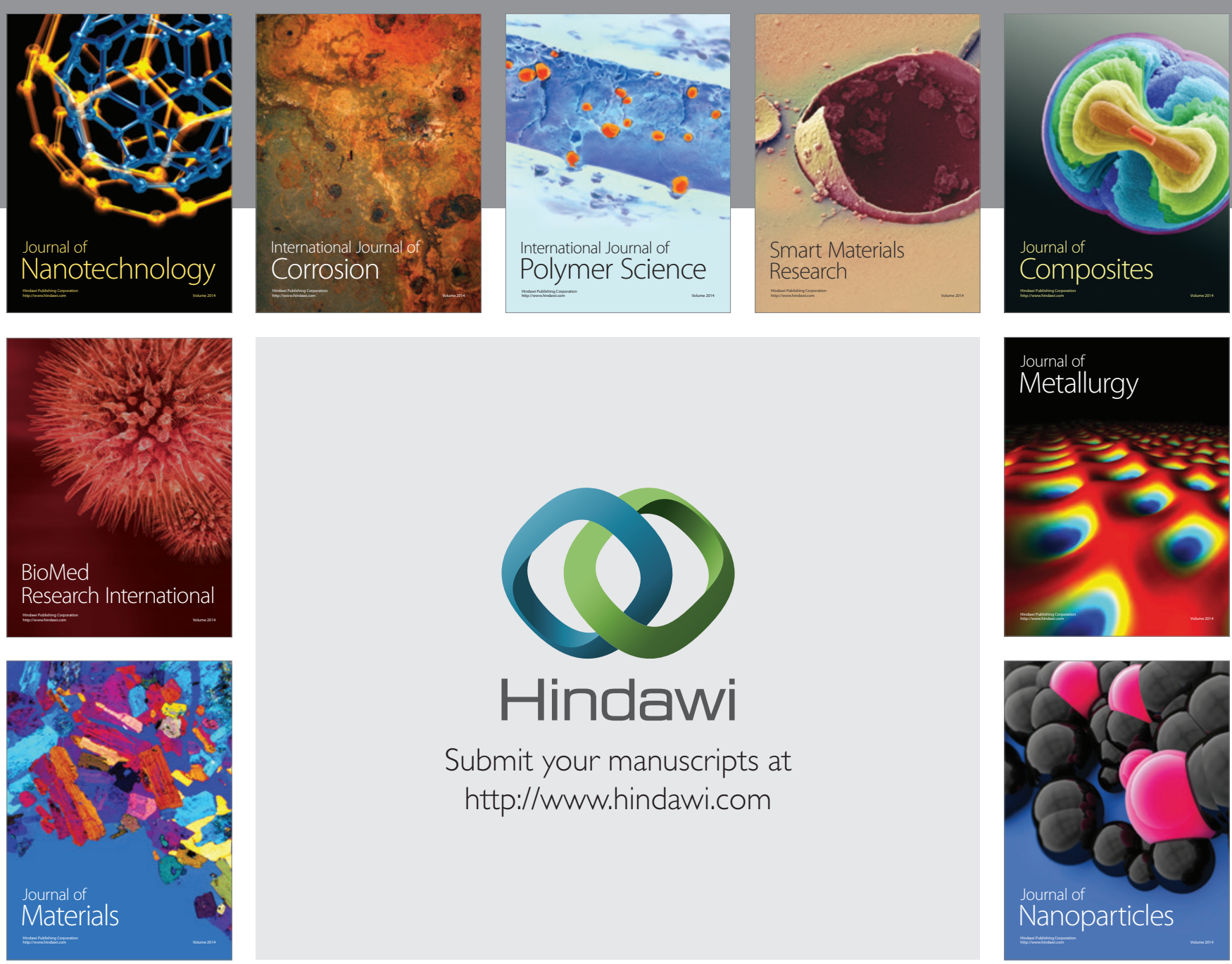

Submit your manuscripts at http://www.hindawi.com
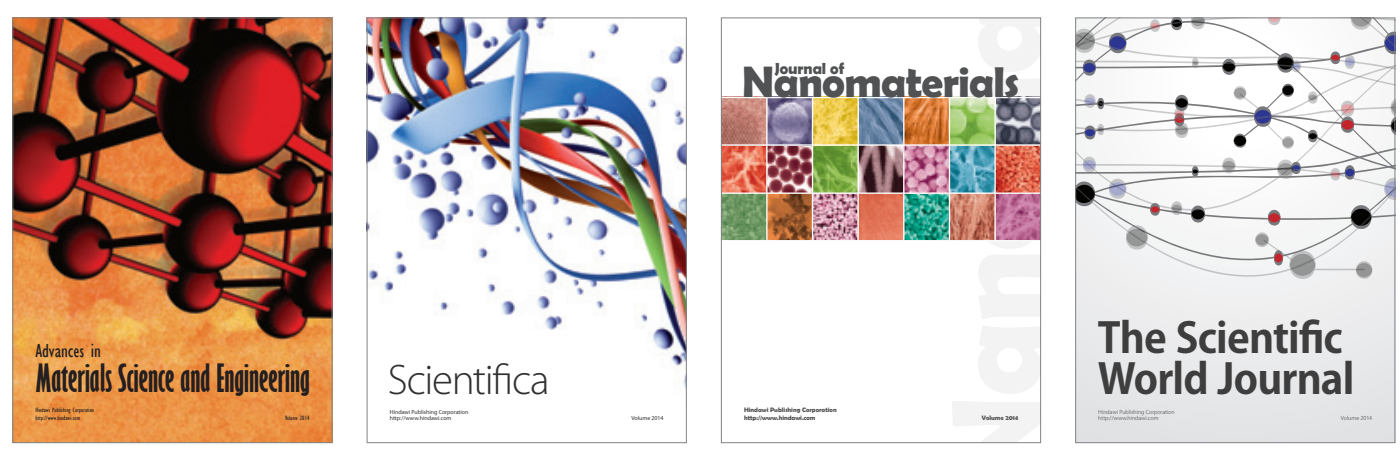

\section{The Scientific World Journal}
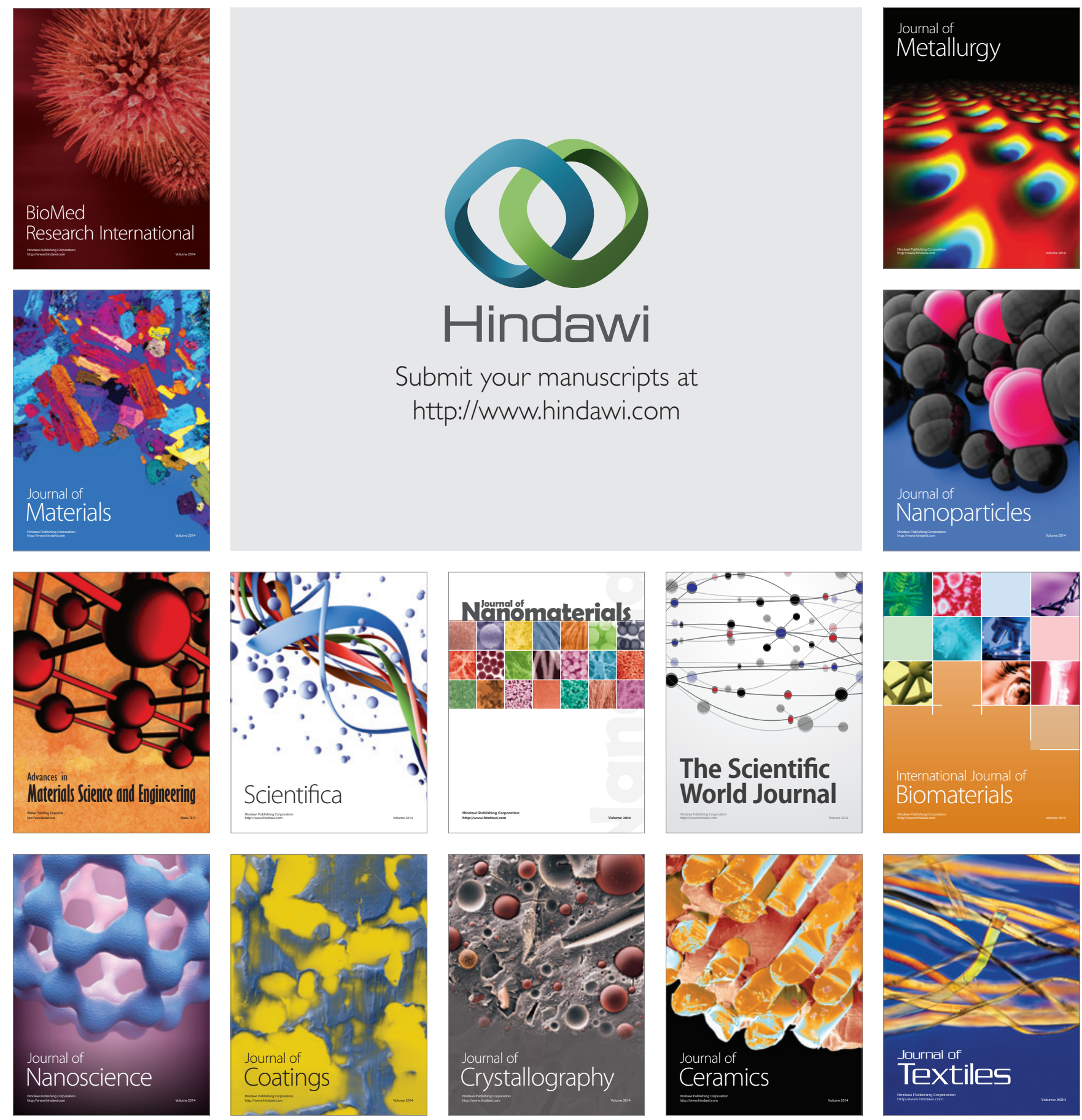